Série des Documents de Travail

\author{
$n^{\circ}$ 2012-09 \\ Inventory Investment \\ and the Business Cycle: \\ The Usual Suspect \\ F. BEC ${ }^{1}$ \\ M. BEN SALEM ${ }^{2}$
}

April 2, 2012

Les documents de travail ne reflètent pas la position du CREST et n'engagent que leurs auteurs. Working papers do not reflect the position of CREST but only the views of the authors.

\footnotetext{
1 THEMA, Université de Cergy-Pontoise, and CREST. Email : bec@ensae.fr

2 Centre d'Etudes de l'Emploi, Paris School of Economics and Université de Paris-Est, France.
} 


\title{
Inventory Investment and the Business Cycle: The Usual Suspect
}

\author{
FRÉDÉRIQUE BEC ${ }^{1}$
}

MÉLIKA Ben SALEM ${ }^{2}$

April 2, 2012

Revised version

\begin{abstract}
From quarterly postwar French data, this paper provides evidence of a bounce-back effect in inventory investment but not in final sales data. Actually, from a bounce-back augmented threshold model, it appears that $i$ ) the null hypothesis of no bounce-back effect is strongly rejected by the inventory investment data and $i$ i) the one-step ahead forecasting performances of the models accounting for this bounce-back effect are well improved compared to linear or standard threshold autoregressions. This supports the conventional wisdom that inventory investment exacerbates aggregate fluctuations, in line with the recent theoretical models by e.g. Wang and Wen [2009] and Wang, Wen and Xu [2011] which clearly predict a destabilizing role of inventory investment over the business cycle. By contrast, our empirical findings cast doubt on models based on the stockouts avoidance motive for holding inventories.
\end{abstract}

Keywords: Inventory investment, Threshold models, bounce-back effects, asymmetric business cycles.

JEL classification: E32, C22.

Acknowledgments: We would like to thank Othman Bouabdallah and Laurent Ferrara, participants at the Computational and Financial Econometrics Conference (London, 2011), the workshop in Honor of Pierre-Yves Hénin (Paris 2012) and the workshop on Nonlinear and Asymmetric Models in Applied Economics (Paris, 2012) as well as two anonymous referees for helpful comments and discussions. We are responsible for all errors and omissions. Frédérique Bec gratefully acknowledges financial support from the Danish Social Sciences Research Council, grant FI-10-079774.

\footnotetext{
${ }^{1}$ THEMA, Université de Cergy-Pontoise, CREST-INSEE, France. email: bec@ensae.fr

${ }^{2}$ Centre d'Etudes de l'Emploi, Paris School of Economics and Université de Paris-Est, France.
} 


\section{Introduction}

Using recent developments in nonlinear time series econometric models, a growing number of empirical works find evidence of a high-growth recovery phase following contractions in real GDP growth rate data (see e.g. Sichel [1994], Kim, Morley and Piger [2005], Bec, Bouabdallah and Ferrara [2011a] or Bec, Bouabdallah and Ferrara [2011b]). To our knowledge, the origins of this bounce-back phenomenon have hardly been explored so far. Yet, a widely held belief points to the inventory investment behavior as a good candidate. As emphasized in Blinder and Maccini [1991]:

"At the macro level, economists have known (but periodically forgotten) since Abramovitz [1950] that inventory movements are dominant features of business cycles. " (p.73)

Indeed, the main stylized facts grounding such a belief are that inventory investment is procyclical and in general slightly positively correlated with sales, while the variance of production is greater than the variance of sales. As a result, the conventional wisdom is that inventory investment exacerbates aggregate fluctuations. Many reasons for holding inventories have been considered in the theoretical literature, and as noticed by Wang and Wen [2009], the destabilizing nature of inventories does not hinge on whether inventory investment is procyclical but on the motives for holding inventories.

Until the eighties, the production smoothing model was dominant. Basically, it relies on the idea that inventories serve as a buffer stock against shocks to demand, but yields the counterfactual implications that production should be less volatile than sales and that inventory investment and sales should be negatively correlated. ${ }^{3}$ Since the mid-eighties, the theoretical research on inventory investment has progressively shifted from an unsatisfactory partial equilibrium approach to a general equilibrium setup and concurrently extended the analysis to other motives for holding inventories. The most prominent ones are $i$ ) the reduction of fixed order costs grounding the so-called $(S, s)$

\footnotetext{
${ }^{3}$ See Blinder [1986] for a detailed presentation of the production smoothing model and how it may be amended to somehow reconcile its implications to the stylized facts.
} 
rule as proposed in Wang et al. [2011] $]^{4}$, ii) the production-costs smoothing as illustrated in Wang and Wen [2009] ${ }^{5}$ and iii) the avoidance of stockouts given demand uncertainty and delay between orders and deliveries ${ }^{6}$. So far, the third motive has not proven successful in generating aggregate output destabilization from a DSGE model, contrary to the first two motives which are crucial to explain how inventory investment may exacerbate business cycles in Wang et al. [2011] and Wang and Wen [2009] DSGE models respectively.

The model proposed by Wang and Wen [2009] retains the production-cost smoothing motive promoted by Eichenbaum [1989], according to which profit-maximizing firms facing cost shocks may choose to "bunch" production by producing more than sales and carrying the excess supply as inventories when costs are low, and using inventories to meet demand when costs are high. Two important predictions of their model are that procyclical inventory investment may i) greatly amplify the volatility of aggregate output and ii) propagate aggregate shocks by generating hump-shaped output dynamics. Hence, this production-cost smoothing model confirms the key role of inventory investment in the business cycle. Another behavior is put forward by Wang et al. [2011] who assume a firm-level $(S, s)$ policy for holding inventories, as in Kahn and Thomas [2007], but augment the model with either a variable capacity utilization rate assumption or capital adjustment costs. Indeed, the non-destabilizing role of inventories result in Kahn and Thomas [2007] directly stems from the fact that a procyclical inventory investment is buffered by a weakened final goods production due to resource reallocation. Consequently, considering a highly "localized" production factor such as the capacity utilization, or mitigating the resource reallocation by imposing adjustment costs, is most likely to offset the smoothing role of sales. The model proposed by Wang et al. [2011]

\footnotetext{
${ }^{4}$ See also Blinder [1981], Blinder and Maccini [1991], Kahn and Thomas [2007]. In the model proposed by the latter, inventories arise as a result of non-convex delivery costs. To economize on such costs, firms hold stocks, making active adjustments only when these stocks are sufficiently far from a target. This behavior grounds the so-called $(S, s)$ rule.

${ }^{5}$ Eichenbaum [1989] has first developed this motive in a partial equilibrium setup.

${ }^{6}$ See Kahn [1987], Bils and Kahn [2000], Kryvtsov and Midrigan [2009], Kryvtsov and Midrigan [2010], Wen [2011].
} 
actually predicts a destabilizing influence of inventories at business cycle frequencies. These two recent theoretical models are compatible with a bounce-back effect in the aggregate output as long as there is one in the inventory investment ${ }^{7}$.

Now there is a nascent empirical evidence of a bounce-back effect in the real GDP growth rate, the theoretical discussion above motivates the empirical investigation of inventory investment dynamics.

Indirect empirical evidence for the inventory investment bounce-back effect was provided in Sichel [1994] from US data. Basically, since the real output is the sum of the final sales and the inventory investment, this author tests for a bounce-back effect in final sales using a very simple regression allowing the average growth rate of the final sales to switch across expansion/contraction/recovery phases over the business cycle. As the lack of bounce-back effect null hypothesis is not rejected for the final sales, whereas it is for the real GDP growth rate, Sichel [1994] concludes that the latter originates in the inventories bounce-back. To our knowledge, no direct test of inventories bounce-back has been performed so far. Our paper aims at filling this gap.

Furthermore, this indirect evidence was obtained from an econometric framework which doesn't allow for endogenous dating of the regime-switches nor for the bounceback magnitude to depend on the duration and/or depth of the recession. Nowadays, more sophisticated nonlinear models may shed new light on the importance of the role played by inventory investment over the business cycle. In particular, we think that the bounce-back augmented threshold autoregressive model proposed recently by Bec et al. [2011b] may be well-suited to capture the kind of dynamics suggested by e.g. Wang and Wen [2009] and Wang et al. [2011] theoretical models. This bounce-back model is an extension of the two-regime self-exciting threshold auto-regression which aims to

\footnotetext{
${ }^{7}$ The idea that firm-level $(S, s)$ policy can spread throughout sectors and/or the whole economy was further explored by Cooper and Haltiwanger [1992] who consider an economy consisting in a retailer for final goods and two manufacturers who produce intermediate goods. They show that a high cost to hold inventories for the manufacturers will imply a production bunching in the manufacturers sector even though it has rising marginal costs: this stems from the bunching of orders by the retail sector as in the $(S, s)$ model.
} 
account for periods of high-growth recoveries following the cycle trough ${ }^{8}$. Moreover, testing for the existence and the shape of the bounce-back effect can be performed quite straightforwardly within this setup.

Hence, our contribution is twofold: $i$ ) it provides a direct test of inventories bounceback, ii) it retains a threshold model which is flexible enough to allow for various bounceback functions to be tested. Using French inventory investment and final sales quarterly growth rates over the period 1950Q2-2011Q1, our estimation results suggest that both the linearity hypothesis and the null of no bounce-back in the threshold model are strongly rejected for inventory investment. By contrast, the linear model cannot be rejected for the final sales data. Moreover, accounting for the bounce-back effect in the threshold model for inventory investment data clearly improves the short-term forecasting performance, and especially so during the recovery episodes. These results are compatible with the fixed order costs and the production-costs smoothing motives for holding inventories, which may explain this bounce-back dynamics in inventories. This in turn may underlie the bounce-back dynamics in the real GDP growth rate.

The paper is organized as follows. Section 1 briefly presents the threshold bounceback ARMA model and discusses the various shapes of bounce-back functions as special cases of the general model. Section 2 describes the data and provides first statistical evidence of inventory investment bounce-back. Section 3 presents the linearity tests before reporting the estimation results. Section 4 evaluates the short-run forecasting performances of the bounce-back models, paying careful attention to contraction/recovery episodes. Section 5 concludes.

\footnotetext{
${ }^{8}$ See Kim et al. [2005] or Morley and Piger [2011] for an extension of the Markov-Switching model which allows bounce-back effects.
} 


\section{The model}

Let $y_{t}$ denote the contribution of change in inventories to the real output growth. The basic model we will consider throughout this paper is the following:

$$
\phi(L) y_{t}=\mu_{t}+\theta(L) e_{t}
$$

with $\mu_{t}$ defined by

$$
\begin{aligned}
\mu_{t}= & \gamma_{0}\left(1-s_{t}\right)+\gamma_{1} s_{t} \\
& +\lambda_{1} s_{t} \sum_{j=\ell+1}^{\ell+m} s_{t-j}+\lambda_{2}\left(1-s_{t}\right) \sum_{j=\ell+1}^{\ell+m} s_{t-j}+\lambda_{3} \sum_{j=\ell+1}^{\ell+m} y_{t-j-1} s_{t-j},
\end{aligned}
$$

and where $\phi(L)$ and $\theta(L)$ are lag polynomials of orders $p$ and $q$ respectively, with roots lying outside the unit circle and $e_{t}$ i.i.d. $\mathcal{N}(0, \sigma) . \ell$ and $m$ are non-negative integers and correspond respectively to the delay with which the bounce-back effect occurs and to its duration. The $\lambda_{i}$ 's parameters measure the size of the bounce-back effect. Let $s_{t}$ denote the transition function which takes on the value zero or one. In our model, $s_{t}$ is defined as:

$$
s_{t}=0 \text { if } y_{t-1}>\kappa \text { and } 1 \text { otherwise, }
$$

where $\kappa$ is a real-valued threshold parameter. The model given by equations (1) to (3) allows for an asymmetric behavior across regimes. Here, $s_{t}=1$ is identified as the low, or contraction regime by assuming $\kappa<0$. It implies that the intercept in equation (1) is $\gamma_{0}$ if the switching variable $y_{t-1}$ is larger than the threshold $\kappa$ (i.e. high, or expansion regime) and $\gamma_{1}$ otherwise. The remainder of equation (2) defines a very flexible form for the bounce-back phenomenon. Indeed it nests the three models first proposed by Kim et al. [2005], namely the U-, V- and Depth-shaped bounce-back ${ }^{9}$ as well as the no bounce-back — standard threshold - model with the following linear restrictions:

- $\mathrm{H}_{0}^{N}: \lambda_{i}=0 \forall i$ corresponds to the standard (no bounce-back) threshold model,

\footnotetext{
${ }^{9}$ See Bec et al. [2011a] and Bec et al. [2011b] for a detailed description of these functions.
} 
- $\mathrm{H}_{0}^{U}: \lambda_{1}=\lambda_{2}=\lambda \neq 0$ and $\lambda_{3}=0$ gives the U-shaped model, hereafter denoted BBU,

- $\mathrm{H}_{0}^{V}: \lambda_{2} \neq 0$ and $\lambda_{1}=\lambda_{3}=0$ gives the BBV model,

- $\mathrm{H}_{0}^{D}: \lambda_{1}=\lambda_{2}=\lambda_{3}=\lambda$ defines the BBD model.

Finally, the general model defined here by equations (1) to (3) will be denoted $\operatorname{BBF}(p, m, \ell)$, as in Bec et al. [2011b]. For $(p, m, \ell)$ parameters assumed known and fixed as described in section 3 below, the BBF model is estimated by the nonlinear least squares method using a grid search on the threshold parameter $\kappa$. The linear null hypothesis amounts here to test the joint hypothesis $\lambda_{1}=\lambda_{2}=\lambda_{3}=0$ and $\gamma_{0}=\gamma_{1}$, i.e. $\mu_{t}$ becomes a constant term. Obviously, the threshold parameter is unidentified under this null. Consequently, the linearity test will rely on a SupLR statistics along the lines proposed by Davies [1987] and its bootstrapped p-value will be computed following Hansen [1996]. Since there are nuisance parameter free, the four assumptions $\mathrm{H}_{0}^{N}, \mathrm{H}_{0}^{U}, \mathrm{H}_{0}^{V}$ and $\mathrm{H}_{0}^{D}$ can be tested from a LR statistics which has a standard Chi-squared distribution.

\section{Data}

The French inventories data used in this analysis come from the INSEE quarterly national accounts database (2005 basis, seasonally adjusted $\left.{ }^{10}\right)$. They correspond to the quarterly contribution of inventory investment to quarterly real GDP growth ${ }^{11}(\times 100)$ over the period 1950Q2-2011Q1. We compute the final sales contribution by substracting the inventory investment contribution series from the real GDP growth series.

Table 1 below reports the average contributions of inventory investment and final sales observed from one to eight quarters after the end of recessions ${ }^{12}$. Regarding the inventory investment series, a bounce-back effect seems to occur from the third quarter

\footnotetext{
${ }^{10}$ Inventory investment is measured by the INSEE as the difference between the national sources and uses other than inventories, namely intermediate consumption, final consumption, gross fixed capital formation and exports.

${ }^{11}$ This series ID number is P54.

${ }^{12}$ According to Bec et al. [2011a], four recessions occurred over the sample under study: 1974Q41975Q2, 1980Q2-1980Q4, 1992Q4-1993Q2, 2008Q2-2009Q3.
} 
following the trough on and lasts four quarters: the same delay and duration are found for the French real GDP growth rate in Bec et al. [2011b], which provides support to the assumption that the bounce-back in real output may originate in inventory investment. Actually, the inventories contributions observed during these four quarters range from $0.31 \%$ to $0.60 \%$, which is well above the average contribution of $0.03 \%$ observed over all expansions. A bounce-back effect of the same duration (four quarters) is found by Sichel [1994] for US inventories data from 1950Q1 to 1992Q4. By contrast, no delay is observed by this author: the US inventories bounce-back activates as soon as the first quarter following the trough. During recessions, the average contribution of inventory

Table 1: Contributions to Real GDP growth

\begin{tabular}{cccc}
\hline \hline $\begin{array}{c}\text { Quarters after } \\
\text { recession }\end{array}$ & $\begin{array}{c}\text { Inventory } \\
\text { contribution } \\
\text { average }\end{array}$ & $\begin{array}{c}\text { Final sales } \\
\text { contribution } \\
\text { average }\end{array}$ & Obs. \\
\hline 1 & 0.016 & 0.257 & 4 \\
2 & -0.347 & $\mathbf{1 . 0 7 1}$ & 4 \\
3 & $\mathbf{0 . 4 4 6}$ & 0.236 & 4 \\
4 & $\mathbf{0 . 5 9 9}$ & 0.285 & 4 \\
5 & $\mathbf{0 . 3 8 6}$ & 0.335 & 4 \\
6 & $\mathbf{0 . 3 1 3}$ & 0.509 & 4 \\
7 & 0.037 & 0.445 & 3 \\
8 & -0.293 & 0.804 & 3 \\
All expansions & 0.035 & 0.865 & 229 \\
All recessions & -0.469 & -0.045 & 15 \\
\hline
\end{tabular}

For 7 and 8 quarters, one observation is lost due to the end of the sample after the last recession.

investment is $-0.47 \%$. Yet, for the same sample and recession dates, it turns out that the average growth rate of French quarterly real GDP is $-0.51 \%$ during recessions. Since only $-0.04 \%$ of this figure comes from the final sales contribution, this suggests that inventory investment accounts for much of the decline in output during contractions a result already found by Sichel [1994] for US data. This is further confirmed by the final 
sales contribution in the quarters following the trough. Looking at the third column of Table 1, it is worth noticing that the contribution of final sales exceeds its average during expansions only once and slightly so: it occurs the second quarter after the trough.

\section{Estimation results}

Following the statistics reported in Table 1, the bounce-back delay parameter $\ell$ and the bounce-back effect duration $m$ are both set to one for the final sales data, while they are respectively set to 2 and 4 for the inventory investment data. The lag lengths of the $\phi(L)$ and $\theta(L)$ polynomials are chosen as the smallest ones which succeed in eliminating residuals serial correlation in the BBF model, i.e. $p=q=1$ for both series.

The SupLR statistics of the linear null hypothesis against a BBF alternative yields quite different conclusions for the series under scrutiny. It strongly rejects the linear null for the inventory investment with a value of 46.16: its bootstrapped $p$-value from 1000 random draws is $1 \%$. The corresponding threshold estimated value is -0.26 . By contrast, the SupLR is only 12.20 from the final sales series, with a bootstrapped $p$-value of $51.6 \%$. Hence, the null of linearity is clearly not rejected in this case, which confirms the result obtained by Sichel [1994] from US final sales data. So as to check the robustness of this result, the linear null is also tested against a BBF alternative in which the real GDP growth rate is the switching variable in equation (3). Again, the linearity is strongly rejected for the inventories whereas it is not for the final sales contribution: the SupLR ( $p$-value) are respectively 51.39 (0.00) and 13.40 (51.2\%). The estimated threshold for the inventory investment contribution is $-0.014 \%$. Altogether, these results provide further evidence that inventory swings are the dominant force in the French GDP swings compared to the final sales. Consequently, the subsequent analysis will focus on the inventory investment only. Table 2 below reports the LR statistics corresponding to the various constrained versions of the BBF model, together with their $p$-values. The top panel corresponds to the Self-Exciting Threshold version of the BBF model, where the switching variable is the contribution of inventories to output growth, whereas 
the bottom panel reports the results obtained when the real GDP growth rate is the switching variable.

Table 2: Testing for the presence and shape of the bounce-back effect

\begin{tabular}{|c|c|c|c|c|c|}
\hline \multirow{3}{*}{$n_{p}$} & $H_{1}: \mathrm{BBF}$ & $H_{0}^{N}:$ no BB & $H_{0}^{U}: \mathrm{BBU}$ & $H_{0}^{V}: \mathrm{BBV}$ & $H_{0}^{D}: \mathrm{BBD}$ \\
\hline & 7 & 4 & 5 & 5 & 7 \\
\hline & \multicolumn{5}{|c|}{ inventory investment as switching variable } \\
\hline Log-Lik & -190.16 & -213.14 & -191.16 & -190.49 & -193.32 \\
\hline LR stat & & 45.96 & 2.00 & 0.66 & 6.32 \\
\hline \multirow[t]{2}{*}{$(p$-val $)$} & & $(0.00)$ & $(0.37)$ & (0.72) & $(0.04)$ \\
\hline & \multicolumn{5}{|c|}{ real GDP growth rate as switching variable } \\
\hline Log-Lik & -180.33 & -190.80 & -181.35 & -182.03 & -187.82 \\
\hline LR stat & & 20.94 & 2.04 & 3.40 & 14.98 \\
\hline$(p$-val $)$ & & $(0.00)$ & $(0.36)$ & $(0.18)$ & $(0.00)$ \\
\hline
\end{tabular}

Again, these results provide support to the bounce-back setup since the standard threshold model, given by $H_{0}^{N}$, is clearly rejected: the null of no bounce-back is rejected at the $1 \%$-level. The D-shaped bounce-back effect is also rejected at conventional level, for both switching variables. By contrast, the BBU and BBV models cannot be rejected according to these results and their estimates are thus presented in Table 3 below.

As can be seen from Table 3, the parameters estimates of these two models are quite similar, whatever the switching variable considered. This is also the case for the Akaike and Schwartz information criteria which are quite close, notwithstanding a very slight domination of the BBV model when the switching variable is the inventories contribution and of the BBU when the switching is governed by lagged GDP growth rate. The only noticeable difference generated by the choice of the switching variable is the percentage of observations lying in each regime: In the case where inventory investment is the switching variable, around $30 \%$ of the observations belong to the low regime, whereas this share falls to nearly $10 \%$ when the transition function depends on the lagged real 
Table 3: Bounce-back threshold models estimates

\begin{tabular}{|c|c|c|c|c|}
\hline \multirow[t]{2}{*}{ switching var. } & \multicolumn{2}{|c|}{ Inventory investment } & \multicolumn{2}{|c|}{ Real GDP growth rate } \\
\hline & $\mathrm{BBU}$ & BBV & $\mathrm{BBU}$ & BBV \\
\hline$\lambda$ & $\mathbf{0 . 0 4}(2.93)$ & $\mathbf{0 . 0 6}(3.17)$ & $\mathbf{0 . 0 6}(4.29)$ & $\mathbf{0 . 0 6}(4.11)$ \\
\hline$\gamma_{0}$ & $0.01(0.48)$ & $-0.02(-0.87)$ & $\mathbf{0 . 0 1}(2.15)$ & $0.01(1.90)$ \\
\hline$\gamma_{1}$ & $-0.18(-3.13)$ & $\mathbf{- 0 . 1 2}(-2.72)$ & $\mathbf{- 0 . 3 1}(-5.20)$ & $\mathbf{- 0 . 2 9}(-4.96)$ \\
\hline$\phi_{1}$ & $\mathbf{0 . 5 1}(5.69)$ & $0.49(5.51)$ & $0.49(7.00)$ & $\mathbf{0 . 4 9}(6.82)$ \\
\hline$\theta_{1}$ & $\mathbf{- 0 . 9 1}(-23.78)$ & $\mathbf{- 0 . 9 0}(-21.89)$ & $\mathbf{- 0 . 9 2}(-29.19)$ & $\mathbf{- 0 . 9 1}(-27.00)$ \\
\hline$\sigma$ & 0.55 & 0.55 & 0.53 & 0.53 \\
\hline$\overline{n_{0}}$ & 159 & 159 & 213 & 213 \\
\hline$n_{1}$ & 77 & 77 & 23 & 23 \\
\hline AIC & 1.655 & 1.650 & 1.579 & 1.585 \\
\hline BIC & 1.728 & 1.723 & 1.652 & 1.658 \\
\hline $\mathrm{Q}(4)[\mathrm{p}$-val $]$ & {$[0.40]$} & {$[0.41]$} & {$[0.25]$} & {$[0.24]$} \\
\hline
\end{tabular}

t-statistics in parenthesis. $\mathrm{Q}($.$) is the Ljung-Box statistics. Bold figures denote$ the $5 \%$ levels. $n_{0}$ (resp. $n_{1}$ ): number of observations in expansion (resp. recession) regime.

GDP growth rate ${ }^{13}$. This discrepancy probably stems from the fact that the inventories contribution variable is much more volatile than the GDP growth rate, and hence is more likely to cross the threshold value than the GDP growth rate. In other words, negative values of the latter are more rare. Figure 1 below reports the corresponding estimated BBU- and BBV- $\mu_{t}$ functions where $s_{t}=1$ two consecutive quarters, which is the estimated average duration in the low regime (top panel) and when $s_{t}=1$ four consecutive quarters (bottom panel): this is the maximal observed duration in this low regime which occurred in 1975Q1 and 1992Q3 in the case where inventory investment is the transition variable and in 1992Q3 and 2008Q3 otherwise. Again, the two competing bounce-back functions display quite similar shapes, especially so for the typical 2-quarter inventory investment contraction. Actually, for such a short-lasting contraction, the U and V-shaped bounce-back functions are quite difficult, if not impossible, to distinguish

\footnotetext{
${ }^{13}$ Notice that the threshold parameter is estimated from a grid search leaving at least $5 \%$ of the observations in the lower regime. Hence, this constraint is not binding.
} 

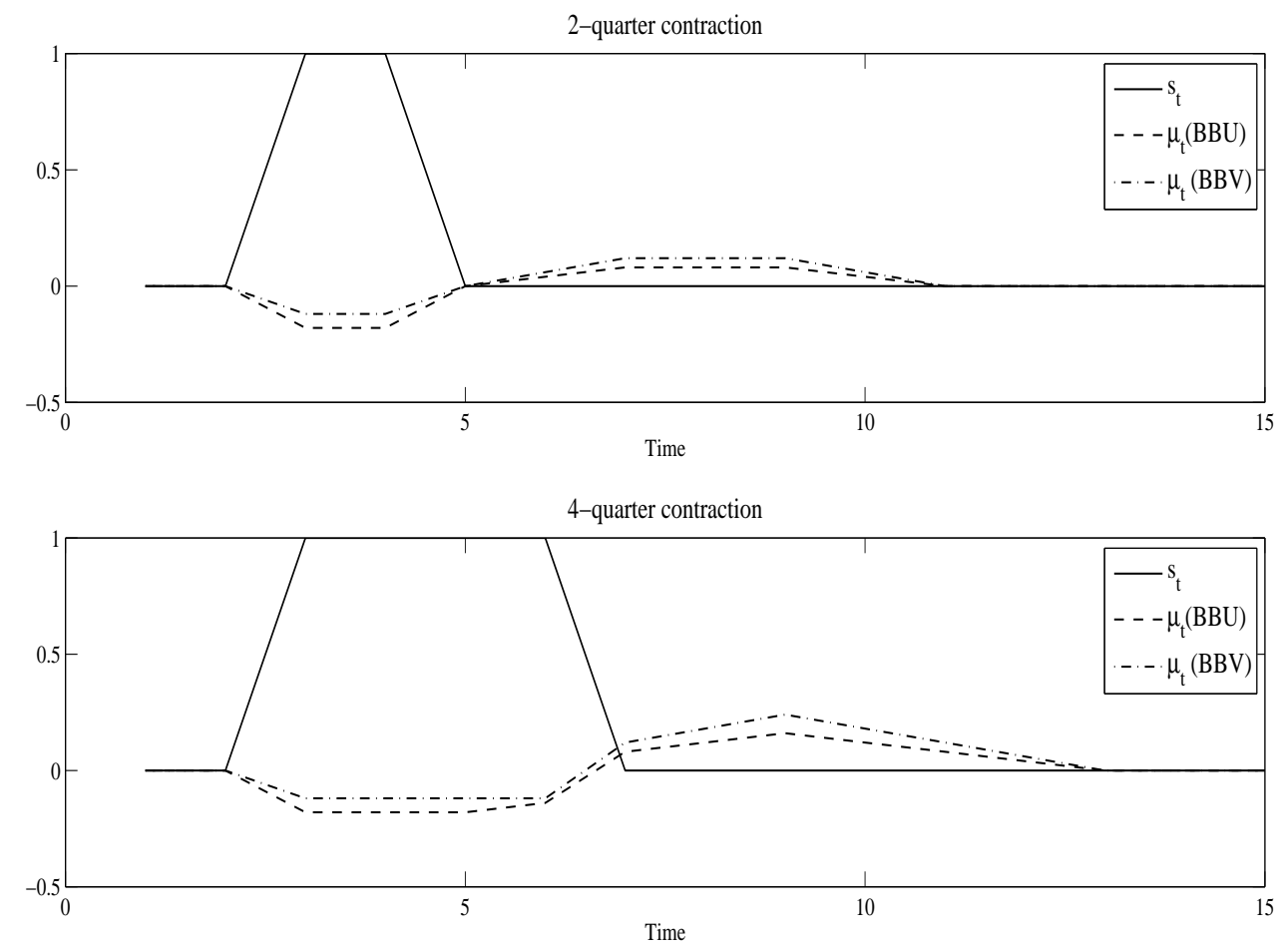

Figure 1: Estimated $\mu_{t}$ functions

statistically because due to the two-quarter delay, the BBU function becomes active after the end of the contraction, in the same way the BBV does by definition. This particularity may explain why these two models provide such close estimation results. By contrast, for a contraction lasting longer, as the four-quarter one, the bounce-back is triggered sooner in the BBU than in the BBV model, as can be seen from the bottom panel of Figure 1.

\section{Short-term forecast performances}

In this section, we focus on the one-step-ahead forecast horizon. Actually, this is the only forecast horizon for which the computation of the optimal forecast in the bounceback threshold model is as straightforward as in the linear model. The first line of Table 
4 reports the conventional Root Mean Squared Errors (RMSE hereafter) criterion computed over the last ten years of the sample. ${ }^{14}$ Its computation relies on a pseudo-real time analysis using recursive regressions: since our final observation date is 2011Q1, we begin the forecast performance evaluation from 2000Q2. Then, for all quarter $t$ between 2000Q2 and 2010Q4, we estimate the model from the initial observation, 1952Q1, until $t$, and use this estimate to compute the one-step-ahead forecasts of the inventory investment contribution. As can be seen from the first line in Table 4, the two bounce-back models clearly outperform the linear autoregression (denoted linear) and the threshold model without bounce-back (denoted no BB) when evaluated according to one-step ahead forecasts over the past decade. The following lines of Table 4 report the same criterion when looking at the forecast behavior of these models during the four identified French recessions. Each episode is further split in two phases: the recession itself (peak from trough) and the recovery episode lasting $m+\ell$ quarters (here 6) after the trough. Again, the bounce-back threshold models almost always outperform the linear and standard threshold models. Expectedly, they behave particularly well during the recovery phase. A closer look at the results further reveals that the BBU model with the real GDP growth rate as the switching variable provides more accurate forecasts than the other BBF models in general. However, regarding the contraction phase, the conclusion is rather mitigated: this model is still the best one during the last contraction episode, but not during the three previous ones. Overall, the results of this exercise emphasize the relevance of the bounce-back models in accounting for the inventory investment dynamics and hence, give further support to our view.

\footnotetext{
${ }^{14}$ We have deliberately chosen not to present Diebold and Mariano [1995] type of tests for the statistical comparison of the predictive accuracy of the different models. First, there are two traditional arguments against their use: i) classical testing with implausible null implies a sizeable small-sample bias in favor of this null and ii) the original forecast comparison, based e.g. on Mean squared Errors, is a strong model selection tool on its own grounds (see amongst others Wei [1992], Inoue and Kilian [2006] or Ing [2007] on this point). Then, as shown in Costantini and Kunst [2011], the small sample bias toward the Diebold-Mariano like null and toward simplicity is especially true when the true DGP is a Threshold Auto-Regression process.
} 
Table 4: One-step ahead forecasts RMSE

\begin{tabular}{l|c|ccc|ccc}
\hline \hline switching var. & & \multicolumn{3}{|c|}{ Inventory investment } & \multicolumn{3}{c}{ Real GDP growth rate } \\
& linear & no BB & BBU & BBV & no BB & BBU & BBV \\
\hline 2000Q2-2011Q1 & 0.399 & 0.428 & 0.382 & 0.379 & 0.362 & $\mathbf{0 . 3 3 4}$ & 0.342 \\
\hline 2000s recession & & & & & & & \\
2008Q2-2011Q1 & 0.510 & 0.536 & 0.498 & 0.493 & 0.436 & $\mathbf{0 . 3 4 4}$ & 0.345 \\
2008Q2-2009Q3 & 0.474 & 0.493 & 0.555 & 0.525 & 0.352 & $\mathbf{0 . 2 1 9}$ & 0.220 \\
2009Q4-2011Q1 & 0.544 & 0.576 & $\mathbf{0 . 4 3 4}$ & 0.458 & 0.506 & $\mathbf{0 . 4 3 4}$ & 0.435 \\
\hline 1990s recession & & & & & & & \\
1992Q4-1994Q4 & 0.413 & 0.421 & 0.403 & 0.377 & 0.518 & $\mathbf{0 . 3 3 5}$ & 0.380 \\
1992Q4-1993Q2 & 0.316 & 0.339 & 0.508 & 0.339 & $\mathbf{0 . 2 0 0}$ & 0.306 & 0.373 \\
1993Q3-1994Q4 & 0.454 & 0.456 & $\mathbf{0 . 3 3 8}$ & 0.395 & 0.619 & 0.349 & 0.384 \\
\hline 1980s recession & & & & & & & \\
1980Q2-1982Q2 & 0.701 & 0.724 & 0.606 & 0.639 & 0.753 & $\mathbf{0 . 5 5 6}$ & $\mathbf{0 . 5 5 6}$ \\
1980Q2-1980Q4 & 0.251 & 0.192 & 0.269 & $\mathbf{0 . 1 7 6}$ & 0.320 & 0.343 & 0.343 \\
1981Q1-1982Q2 & 0.840 & 0.876 & 0.718 & 0.773 & 0.894 & $\mathbf{0 . 6 3 7}$ & $\mathbf{0 . 6 3 7}$ \\
\hline 1970s recession & & \multicolumn{7}{|c|}{1.876} & & & \\
1974Q4-1977Q1 & 1.02 & 1.01 & 1.16 & $\mathbf{0 . 9 4 8}$ & 1.31 & 1.01 & 1.01 \\
1974Q4-1975Q2 & 1.49 & 1.43 & 1.87 & $\mathbf{1 . 3 3}$ & 1.96 & 1.66 & 1.66 \\
1975Q3-1977Q1 & 0.739 & 0.760 & 0.656 & 0.728 & 0.898 & $\mathbf{0 . 5 2 0}$ & $\mathbf{0 . 5 2 0}$ \\
\hline
\end{tabular}

Note: Bold characters denote the smallest RMSE.

\section{Concluding remarks}

This paper shows from empirical grounds the existence of a bounce-back effect in French inventory investment data which may be the cause of the bounce-back effect found by Bec et al. [2011b] for the French real GDP growth rate. Actually, two reasons suggest the key role of inventory investment in aggregate fluctuations: first, no such bounceback effect appears in the final sales data and second, the delay and duration of the bounce-back found in the inventory investment data are the same as the ones estimated by Bec et al. [2011b] for the output growth rate. These empirical results provide support to the theoretical models proposed recently by Wang and Wen [2009] and Wang et al. [2011] which clearly predict that inventory investment may greatly amplify the volatility 
of aggregate output. By contrast, our conclusions cast doubt on models based on the stockouts avoidance motive for holding inventories since so far, they predict a stabilizing or neutral role of inventories in the business cycle. Our results have to be extended in at least two ways. First, we need to check that they also hold in other countries. Then, the estimation of a Threshold Vector AutoRegression with bounce-back effect to describe the joint dynamics of inventories and real GDP is on our research agenda. 


\section{References}

Abramovitz, M., Inventories and Business Cycles, New York: National Bureau of Economic Research, 1950.

Bec, F., O. Bouabdallah, and L. Ferrara, The possible shapes of recoveries in Markov switching models, Working Paper 2011-02, CREST 2011a.

_, _, and _ _ The European Way Out of Recessions, Manuscript 2011b.

Bils, M. and J. Kahn, What Inventory Behavior Tells Us about Business Cycles, The American Economic Review, 2000, 90 (3), 458-481.

Blinder, A.S., Retail Inventory Behavior and Business Fluctuations, Brookings Papers on Economic Activity, 1981, 2, 443-505.

_ Can the Production Smoothing Model of Inventory Behavior Be Saved, Quarterly Journal of Economics, 1986, 101 (3), 431-453.

— a and L.J. Maccini, Taking Stock: A Critical Assessment of Recent Research on Inventories, Journal of Economic Perspectives, 1991, 5 (1), 73-96.

Cooper, R. and J. Haltiwanger, Macroeconomic implications of production bunching : Factor demand linkages, Journal of Monetary Economics, 1992, 30 (1), 107-127.

Costantini, M. and R. Kunst, On the Usefulness of the Diebold-Mariano Test in the Selection of Prediction Models: Some Monte Carlo Evidence, Working Paper 276, Institute for Advanced Studies, Vienna 2011.

Davies, R. B., Hypothesis Testing When a Nuisance Parameter is Present Only Under the Alternative, Biometrika, 1987, 74, 33-43.

Diebold, F.X. and R.S. Mariano, Comparing Predictive Accuracy, Journal of Business and Economic Statistics, 1995, 13 (3), 253-263. 
Eichenbaum, M., Some Empirical Evidence on the Production Level and Production Cost Smoothing Models of Inventories, American Economic Review, 1989, 79 (4), 853-864.

Hansen, B.E., Inference when a Nuisance Parameter Is Not Identified Under the Null Hypothesis, Econometrica, 1996, 64 (2), 413-430.

Ing, C.K., Accumulated prediction errors, information criteria and optimal forecasting for autoregressive time series, Annals of Statistics, 2007, 35, 12381277.

Inoue, A. and L. Kilian, On the selection of forecasting models, Journal of Econometrics, 2006, 130, 273306.

Kahn, J., Inventories and the Volatility of Production, The American Economic Review, 1987, 77 (4), 667-679.

_ and J. Thomas, Inventories and the Business Cycle: An Equilibrium Analysis of (S,s) Policies, The American Economic Review, 2007, 97 (4), 1169-1188.

Kim, C.-J., J. Morley, and J. Piger, Nonlinearity and the permanent effects of recessions, Journal of Applied Econometrics, 2005, 20, 291-309.

Kryvtsov, O. and V. Midrigan, Inventories, Markups, and Real Rigidities in Menu Cost Models, Working Paper 14651, NBER 2009.

_ els, Journal of The Japanese and International Economies, 2010, 24, 259-281.

Morley, J. and J. Piger, The asymmetric business cycle, Review of Economics and Statistics, 2011.

Sichel, E., Inventories and the Three Phases of the Business Cycles, Journal of Business and Economic Statistics, 1994, 12 (3), 269-277. 
Wang, P. and Y. Wen, Inventory Accelerator in General Equilibrium, Working Paper 010, Federal Reserve Bank of St. Louis 2009.

—, _ , and Z. Xu, When Do Inventories Destabilize the Economy? An Analytical Approach to (S,s) Policies, Working Paper 014, Federal Reserve Bank of St. Louis 2011.

Wei, C.Z., On predictive least squares principles,, Annals of Statistics, 1992, 20, 1-42.

Wen, Y., Input and Output Inventory Dynamics, American Economic Journal: Macroeconomics, 2011, 3, 1-33. 


\section{Appendix}

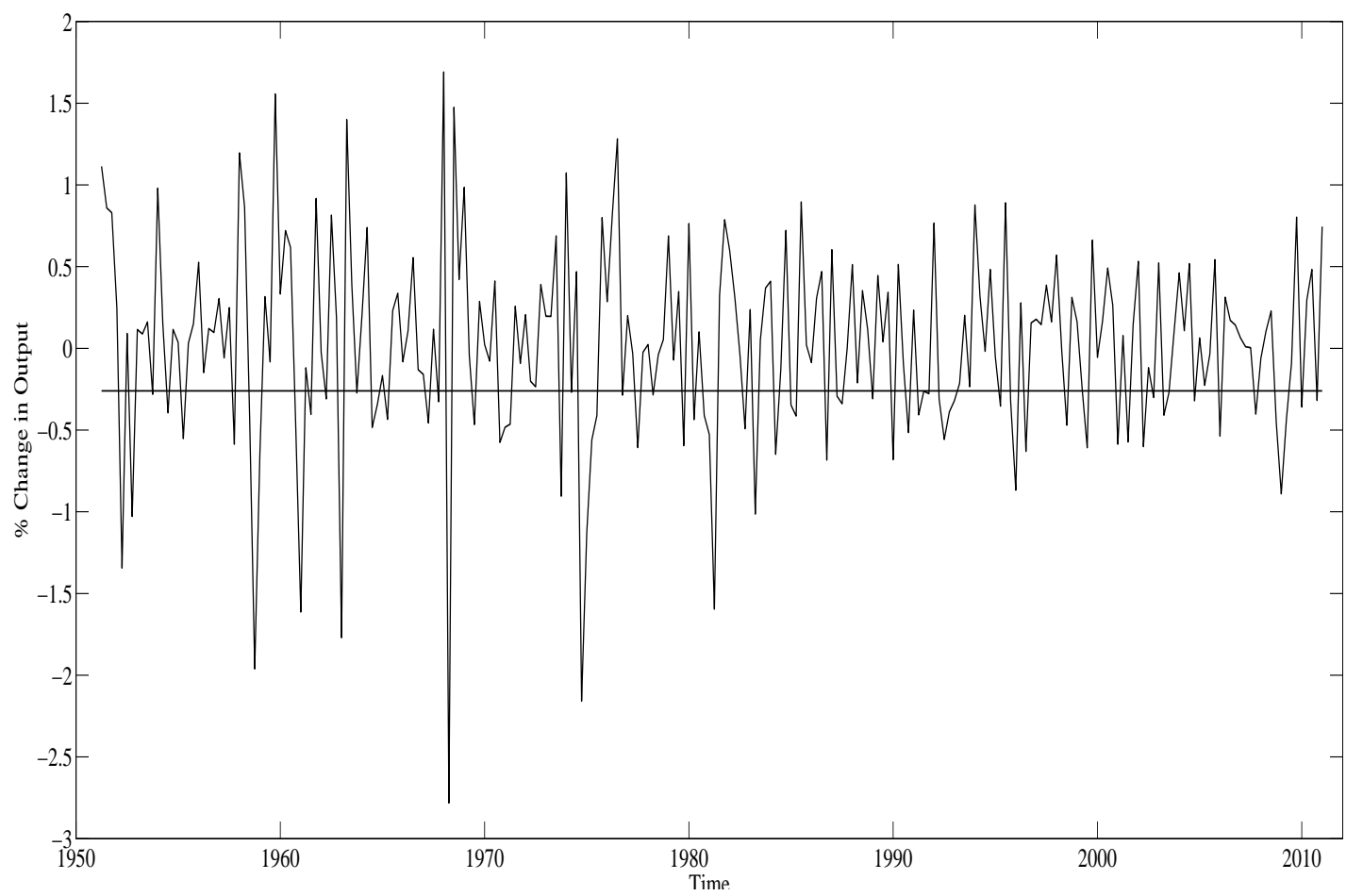

Figure 2: Inventories contribution data and estimated thresholds 